\title{
LA IMPORTANCIA DEL TRABAJO BOTÁNICO DE AIMÉ BONPLAND EN SUDAMÉRICA Y LA INCÓGNITA DE LAS COLECCIONES BOTÁNICAS REALIZADAS EN PARAGUAY
}

\section{The importance of Aimé Bonpland's botanical work in South America and unknown of the botanical collections made in Paraguay}

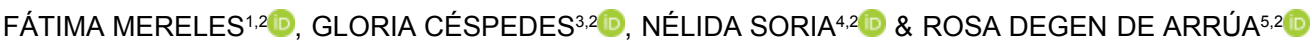

\begin{abstract}
Resumen: Se presenta una síntesis del estado de conocimiento que se tiene en Paraguay sobre la vida y obra de Aimé Bonpland, quien vivió en el país cerca de 10 años. Como objetivos se resaltan la importancia de su trabajo como botánico taxónomo, su interés en el uso de las plantas en general, además de dedicarle un sencillo homenaje. Para ello se recurrió a la bibliografía especializada, se consultaron bases de datos en línea, los fascículos de la Flora del Paraguay y se revisaron los herbarios nacionales. Como resultado se da a conocer una lista no exhaustiva de las especies que determinó taxonómicamente y que están presentes en la flora paraguaya y como homenaje a su legado, se complementa con una lista de los géneros y epítetos de las especies de plantas vasculares registradas para la flora paraguaya que les fueran dedicados. El trabajo concluye con la incógnita de la cantidad y el destino de las especies que fueron colectadas en el Paraguay.
\end{abstract}

Palabras clave: Aimé Bonpland, colecciones botánicas, Corrientes, Paraguay, plantas útiles, taxonomía.

\begin{abstract}
Summary: A synthesis of the state of knowledge that exist in Paraguay about the life and work of Aimé Bonpland, who lived in the country about 10 years, is here presented. The objectives were to highlight the importance of his work as a plant taxonomist and his interest in the use of plants in general, in addition to dedicate him a simple tribute. For all this, the specialized bibliography, online databases and fascicles of the Flora of Paraguay were consulted, and national herbaria were revised. As a result, two lists were compiled: a non-exhaustive list of the species he determined species in the Paraguayan flora and, as a tribute to its legacy, a list of the genera and epithets of the vascular plant species of the Paraguayan flora that were dedicated to him. This work concludes with the unknown quantity and destination of species collected in Paraguay.
\end{abstract}

Key words: Aimé Bonpland, botanical collections, Corrientes, Paraguay, taxonomy, useful plants.

\section{Introducción}

Los nombres de Alexander von Humboldt y Aimé Bonpland (conocido también como don
Amado), son frecuentemente mencionados en Biología, mucho más el de Humboldt, el gran maestro de las ciencias naturales y en particular de la ecología y fitogeografía. Con

\footnotetext{
${ }^{1}$ Centro para el Desarrollo de la Investigación Científica (CEDIC). Fundación Moisés Bertoni \& Laboratorios DíazGill. Asunción, Paraguay. E-mail: fmereleshaydar@gmail.com

${ }^{2}$ Programa Nacional de Incentivo a Investigadores, Consejo Nacional de Ciencia y Tecnología, CONACYT, Asunción.

${ }^{3}$ Facultad de Ciencias Agrarias, Universidad Nacional de Concepción, Paraguay. E-mail: gloriacespedes@gmail.com

${ }^{4}$ Facultad de Ciencias Aplicadas, Universidad Nacional de Pilar, Paraguay. E-mail: nsoria2000@yahoo.com

${ }^{5}$ Facultad de Ciencias Químicas, Universidad Nacional de Asunción, Paraguay. E-mail: rdegen@qui.una.py
} 
un legado muy grande, Humboldt colaboró con la descripción de las formas de la tierra firme, la climatología, la hidrografía, incluidas las corrientes oceanográficas, la fitogeografía, que constituye probablemente su acercamiento a la botánica y la geografía de los animales, entre otras cuestiones (Beck et Schoenwalt, 1999).

En su gran viaje a las regiones equinocciales, Humboldt fue acompañado por Aimé Jacques Alexandre Goujard-Bonpland llamado simplemente Aimé Bonpland o Amado Bonpland, como se lo conoce en Argentina y Paraguay, quien sentía una particular afección por el conocimiento de otras ramas de las ciencias naturales como la botánica (Boccia Romañach, 2001) y ya manifiesta su interés en dicha ciencia cuando a pesar de haber estudiado medicina, concurría a las clases de René Desfontaine y Bernard de Jussieu en el Museo de Ciencias Naturales de París, haciendo de las plantas la pasión de su vida (Lourteig, 1977). La misma autora afirmaba que los dos naturalistas no se parecían, pero se complementaban por el gran amor hacia la naturaleza y la sólida educación de ambos.

La ciencia botánica se encontraba en Europa entonces en pleno apogeo desde la década de 1750 con el equipo de Carl von Linneo, en la de 1760 con Jean Baptiste D’Arnault, quien estudiaba la "nuez moscada" y la "canela" de Venezuela y Charles-Marie de La Condamine quien publicaba sus estudios sobre la "quina". En la década de 1770, Hipólito Ruíz y José Pavón, ya realizaban la Real Expedición al Virreinato del Perú y en la década de 1780, José Celestino Mutis estudiaba las plantas del Virreinato de Nueva Granada, hoy Colombia (Mereles, 2008). De manera que cuando ambos naturalistas investigadores llegaron al nuevo mundo, la ciencia ya estaba en desarrollo y tomaron contacto con sus colegas, tanto con J. C. Mutis como con Juan José de Caldas.

Es interesante mencionar que en materia de estudios botánicos regionales en América, ya en 1799 se publicaba la Flora Huayaquilensis (o flora de la actual Guayaquil, Ecuador), como resultado de la expedición de Juan José Tafalla al extremo litoral del Ecuador y en 1806 José Mejía Lequerica publicaban Las Plantas Quiteñas. Esta última, del área conocida hoy como Ecuador y que evidentemente la misma se constituyó en un atractivo especial, ya que esta parte del mundo, a pesar de su escasa superficie, presenta un gran número de especies vegetales, resultado de sus diferentes tipos de hábitats y es considerado uno de los países mega diversos de Latinoamérica (Mereles, 2008).

En Europa, Bonpland se relacionó con los grandes maestros botánicos de la época: André Thouin, quien dictó numerosos cursos y publicó, entre otras obras "Manuel d'arboriculture", Jean Baptiste de Monet, caballero de Lamarck, quien contribuyó a la flora francesa con su "Flore Françoise" en 1778 y otros estudiosos de la botánica de la época como De Candolle, y Poiret con su "Encyclopèdie Mèthodique Botanique", 1783-1817. Todos estos botánicos mencionados traían consigo los conocimientos acabados del equipo de Carl von Linneo desde 1750. En el mismo siglo iniciaron su aparición los primeros Jardines Botánicos en Italia, con cultivos de especies vegetales nativas y exóticas llevadas al viejo mundo por diferentes exploradores (Mereles, 2008).

En Paraguay, al igual que en muchos otros lugares, el nombre de Alexander von Humboldt es muy conocido, especialmente por los biólogos e historiadores, entre otros. A pesar de lo mucho que se ha escrito sobre ambos naturalistas, el nombre de Amado Bonpland en Paraguay fue y es increíblemente ignorado, al contrario del grado de conocimiento que tiene el pueblo cubano sobre la figura de A. von Humboldt, su compañero de viaje a América, tal como lo manifiesta Prüfer Leske (2019). La excepción en Paraguay corresponde a personas que de alguna manera han tenido acceso a la literatura biológica e histórica y cuyos resultados son algunos escritos producidos por historiadores acerca de este personaje (Boccia Romañach, 1999, 2001; Contreras Roqué et Boccia Romañach, 2006; entre otros).

Esto llama mucho la atención porque Bonpland vivió en Paraguay más de nueve años en los alrededores del pueblo de Santa María de Fe, departamento de Misiones. El desconocimiento también puede deberse a que Bonpland si bien no estaba detenido, estaba confinado a dicho sitio, aunque se movía a departamentos vecinos como Itapúa, por ejemplo, comercializando sus productos. 
Por otra parte, la historia de casi 10 años de vida de Bonpland en Paraguay tuvo su origen en una reacción del Dr. Don José Gaspar Rodríguez de Francia, dictador supremo del Paraguay y que culminó con el apresamiento del personaje. La vida un poco particular del dictador Rodríguez de Francia fue siempre un gran atractivo para los historiadores, motivo por el cual en Paraguay a Bonpland se 1o recuerda más por el lado de la Historia y su relación con don J. G. Rodríguez de Francia que por el lado de las ciencias naturales.

Otra razón de la falta de bibliografía sobre Bonpland puede deberse a que el Paraguay sufrió dos guerras muy importantes por sus terribles consecuencias, con apenas 50 años de diferencia, en donde posteriormente los historiadores tomaron ambos eventos como grandes temas de investigación. En cuanto a los biólogos/botánicos, se tiene muy escasa referencia bibliográfica (Bordas, 1990; Boccia Romañach, 1999, 2001; Schneppen, 1999; Contreras Roqué et Boccia Romañach, 2006; Mereles, 2008; Sanjurjo, 2008, 2019), conocidos hasta el momento. Se agregan algunas conferencias dadas a iniciativa de la Alianza Francesa de Asunción, por la Prof. Dra. Eugenia Bordas y el Prof. Ing. Ftal. Marcos Sanjurjo, como conferencistas (Fig. 1), algunas reuniones en Santa María de Fe, a iniciativa conjunta de las Embajadas de Alemania y Francia, con el apoyo del Instituto Cultural Paraguayo-Alemán, "Goethe Zentrum" y con la participación de la comunidad científica, muy reducida por cierto.

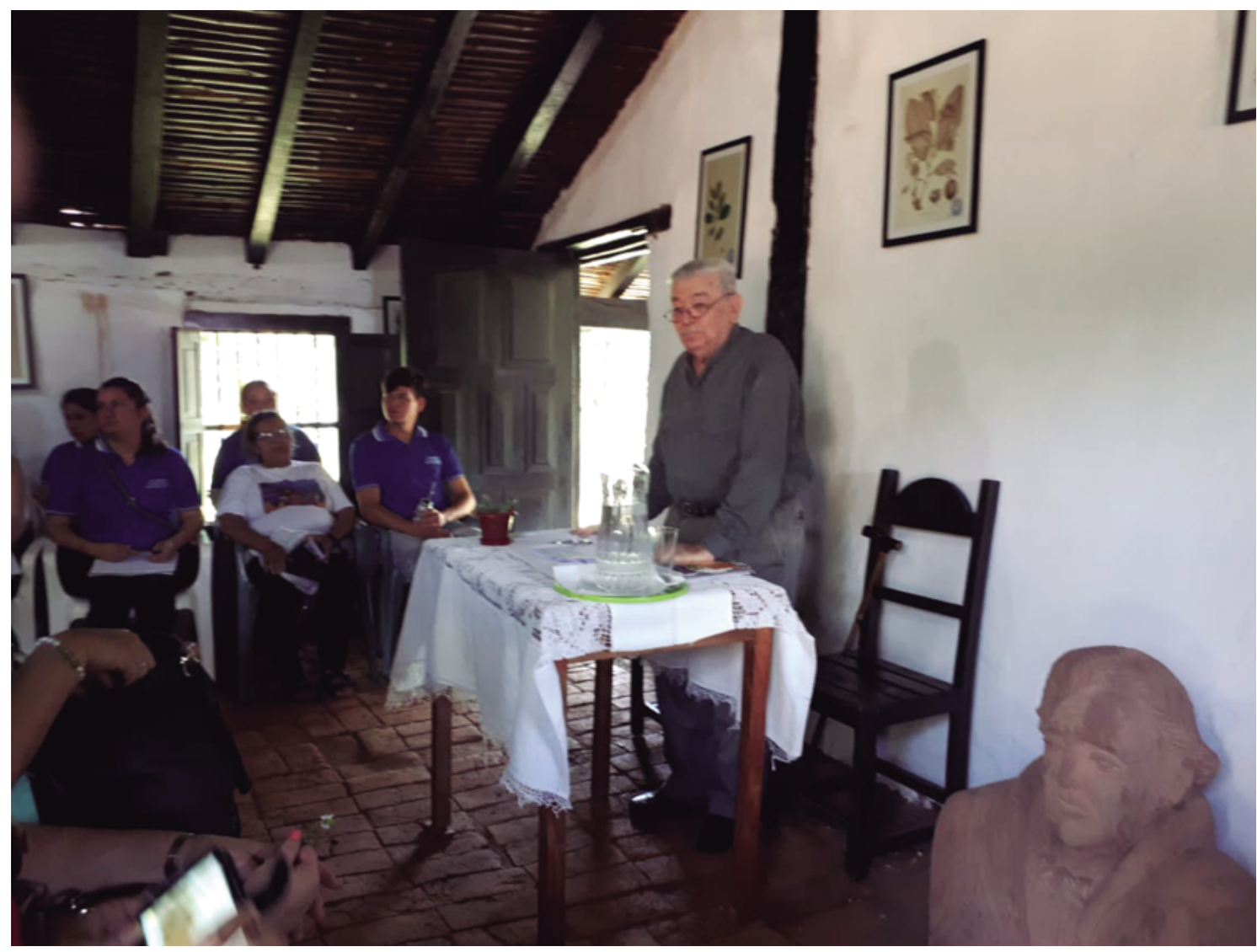

Fig. 1. Conferencia sobre Bonpland en Santa María de Fe, Paraguay (fotografía proporcionada por Rosa Degen de Arrúa).

Fig. 1. Bonpland conference in Santa María de Fe, Paraguay (photograh provided by Rosa Degen de Arrúa). 
En los últimos años, a iniciativa de la embajada de Francia en el Paraguay y con el apoyo del Instituto de Cultura, la Asociación Cultural Bonpland de la ciudad de Corrientes, la Sociedad Científica del Paraguay y la Alianza Francesa de Asunción, se realizó una exposición sobre la vida y la obra de A. Bonpland en Asunción y Santa María de $\mathrm{Fe}$, con la participación de la comunidad local y cuya Escuela de Educación Primaria Básica lleva el nombre de Amado Bonpland. Probablemente esta iniciativa dio pie a la siguiente mención de un evento en Santa María de Fe.

En efecto, mención especial merece la iniciativa del exembajador francés en Paraguay Denis Véne, quien adquirió una de las residencias más antiguas del pueblo de Santa María de Fe y para tener presente en la memoria de la comunidad la denominó Casa Bonpland, que guarda entre otras cosas un jardín de aclimatación de plantas medicinales (Fig. 2A) y un busto del botánico, inaugurado en el evento (Fig. 2B).

Finalmente, un evento reciente a iniciativa de la Asociación Internacional HumboldtBonpland, en el año 2019 se realizó en la Sociedad Científica del Paraguay en Asunción el III Congreso Internacional e Interdisciplinar Humboldt-Bonpland, con el apoyo de las embajadas de Alemania y Francia y el Consejo Nacional de Ciencia y Tecnología, CONACYT, con la participación de numerosos conferencistas internacionales y conocedores de nivel sobre ambos personajes.

Lamentablemente una vez más, el evento mencionado no tuvo el resultado esperado por parte de la organización paraguaya, ya que la participación local en la presentación de los trabajos fue muy escasa y la asistencia del público local nuevamente se circunscribió a gente con bastante conocimiento sobre ambos personajes y prácticamente sin la participación tanto de la comunidad científica como tampoco la de docentes del cuerpo de enseñanza primaria y media, el que fue uno de los grandes objetivos, pues son personas clave en la difusión del conocimiento, especialmente a los niños y jóvenes.

Los objetivos de este trabajo son resaltar la importancia del trabajo de A. Bonpland como botánico, presentando una lista no exhaustiva de las especies que determinó taxonómicamente y que están presentes en la flora paraguaya, resaltar su interés en el uso de las plantas en general y presentar como un homenaje a su legado, una lista de los géneros y epítetos de las familias de plantas vasculares registradas para la flora paraguaya, que les hayan sido dedicados.

\section{Materiales y Métodos}

Para la elaboración del listado de especies descriptas por Bonpland (Tabla 1), como las dedicadas a él (Tabla 2), se recurrió a:

Los fascículos de la Flora del Paraguay.

Las adendas a la Flora del Paraguay publicadas en la revista Candollea: Notulae ad Floram Paraquiensem.

Las bases de datos:

$\mathrm{https}$ ://science.mnhn.fr/all/list?recordedBy=Bonpland

http://www.darwin.edu.ar/Proyectos/FloraArgentina/DetalleEspecie http://www.ipni.org/

http://www.theplantlist.org/

https://www.tropicos.org/Name

http://www.ville-ge.ch/musinfo/bd/cjb/chg/index.php?lang=fr

La Flora del Paraguay aún no está completa, por lo tanto, se recurrió al material de herbario de las familias no publicadas, existentes en los siguientes herbarios nacionales: FCQ, PY (siglas según Thiers, 2020) y el Herbario Histórico de la Sociedad Científica del Paraguay (SCPY), para tratar de completar la información.

Se incluyeron los epítetos válidos y los sinónimos.

\section{Resultados}

Evidentementeel número de determinaciones taxonómicas de las colectas realizadas por Humboldt y Bonpland, reflejan un gran trabajo realizado por el último. Algunas de estas especies se encuentran presentes en Paraguay, como el caso de las 19 listadas en la Tabla 1.

En la Tabla 2 se enumeran 11 especies que están registradas para Paraguay y que les han sido dedicadas. 

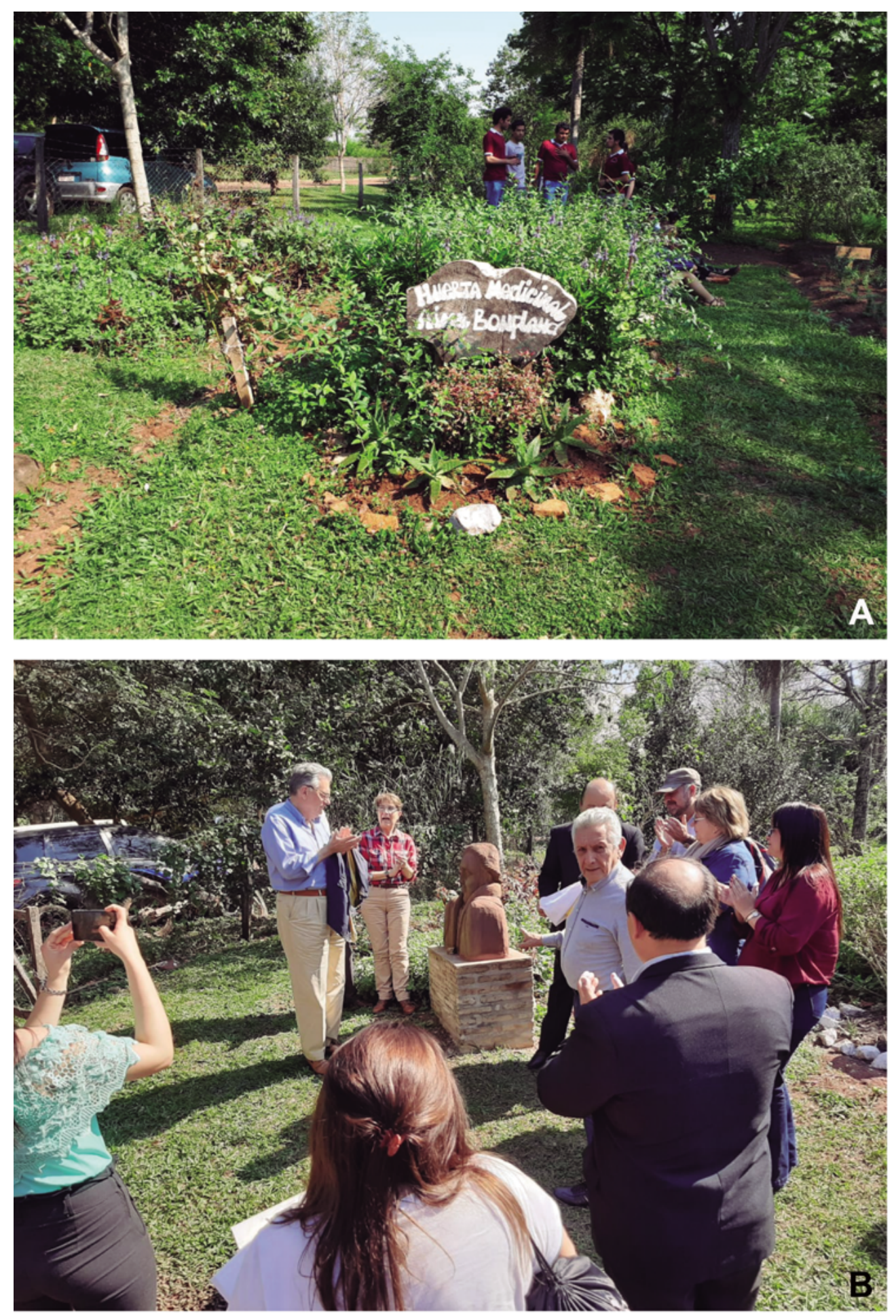

Fig. 2. Fotografías de la Casa Bonpland en Santa María de Fe, Paraguay. A: Jardín de plantas medicinales Aimé Bonpland. B: Inauguración del busto de Aimé Bonpland (fotografías proporcionadas por Rosa Degen de Arrúa).

Fig. 2. Photographs of the Bonpland House in Santa María de Fe, Paraguay. A: Aimé Bonpland medicinal plant garden. B: Inauguration of the Aimé Bonpland bust (photograhs provided by Rosa Degen de Arrúa). 
Tabla 1. Colaboración de Aimé Bonpland en descripción de especies presentes en Paraguay.

Table 1. Colaboration of Aimé Bonpland in the descriptions of species present in Paraguay.

\begin{tabular}{|c|c|c|c|c|c|}
\hline $\mathbf{N}^{\circ}$ & Familia & Especie & Publicación & Status & Observación \\
\hline 1 & Amaranthaceae & $\begin{array}{l}\text { Guilleminea } \\
\text { densa (Humb. } \\
\text { \& Bonpl. ex } \\
\text { Schult.) Moq. }\end{array}$ & $\begin{array}{l}\text { Prodromus Systematis } \\
\text { Naturalis Regni } \\
\text { Vegetabilis } 13(2): 338 . \\
1849 .\end{array}$ & Actual & $\begin{array}{l}\text { Basónimo: } \\
\text { Illecebrum densum } \\
\text { Humb. \& Bonpl. ex } \\
\text { Willd. }\end{array}$ \\
\hline 2 & Apocynaceae & $\begin{array}{l}\text { Ruehssia } \\
\text { macrophylla } \\
\text { (Humb. \& } \\
\text { Bonpl. ex } \\
\text { Schult.) H. } \\
\text { Karst. }\end{array}$ & $\begin{array}{l}\text { Verhandlungen des } \\
\text { Vereins zur Beförderung } \\
\text { des Gartenbaues in den } \\
\text { Königlich Preussischen } \\
\text { Staaten 19: 305. 1849. }\end{array}$ & Actual & $\begin{array}{l}\text { Basónimo: } \\
\text { Asclepias } \\
\text { macrophylla } \\
\text { Humb. \& Bonpl. ex } \\
\text { Schult. }\end{array}$ \\
\hline 3 & Asteraceae & $\begin{array}{l}\text { Vernonia } \\
\text { rubricaulis } \\
\text { Bonpl. var. } \\
\text { pseudoincana } \\
\text { Hieron. }\end{array}$ & $\begin{array}{l}\text { Bot. Jahrb. Syst. 22: } 689 . \\
1897 .\end{array}$ & $\begin{array}{l}\text { Sinónimo de } \\
\text { Lessingianthus } \\
\text { rubricaulis } \\
\text { (Bonpl.) H. Rob. }\end{array}$ & \\
\hline 4 & Asteraceae & $\begin{array}{l}\text { Vernonia } \\
\text { rubricaulis } \\
\text { Bonpl. }\end{array}$ & $\begin{array}{l}\text { Plantae Aequinoctiales } \\
\text { 2(11-13): 66, t. } 99 . \\
\text { 1809[1811]. }\end{array}$ & $\begin{array}{l}\text { Sinónimo de } \\
\text { Lessingianthus } \\
\text { rubricaulis } \\
\text { (Bonpl.) H. Rob. }\end{array}$ & \\
\hline 5 & Asteraceae & $\begin{array}{l}\text { Mikania guaco } \\
\text { Bonpl. }\end{array}$ & $\begin{array}{l}\text { PI. Aequinoct. 2(13): } 84 \text {, } \\
\text { t. } 105.1809 \text { [1811]. }\end{array}$ & Actual & \\
\hline 6 & Bignoniaceae & $\begin{array}{l}\text { Arrabidaea } \\
\text { chica (Bonpl.) } \\
\text { B. Verl. }\end{array}$ & $\begin{array}{l}\text { Revue Horticole (Paris) } \\
\text { 40: } 154.1868 .\end{array}$ & $\begin{array}{l}\text { Sinónimo de } \\
\text { Fridericia chica } \\
\text { (Bonpl.) L.G. } \\
\text { Lohmann }\end{array}$ & \\
\hline 7 & Euphorbiaceae & $\begin{array}{l}\text { Alchornea } \\
\text { castaneifolia } \\
\text { (Humb. \& } \\
\text { Bonpl. ex } \\
\text { Willd.) A. Juss. }\end{array}$ & $\begin{array}{l}\text { De Euphorbiacearum } \\
\text { Generibus Medicisque } \\
\text { earumdem viribus } \\
\text { tentamen, tabulis aeneis } \\
18 \text { illustratum } 42.1824 .\end{array}$ & Actual & $\begin{array}{l}\text { Basónimo: } \\
\text { Hermesia } \\
\text { castaneifolia } \\
\text { Humb. \& Bonpl. ex } \\
\text { Willd. }\end{array}$ \\
\hline 8 & Fabaceae & $\begin{array}{l}\text { Centrosema } \\
\text { sagittatum } \\
\text { (Humb. } \\
\text { \& Bonpl. } \\
\text { ex Willd.) } \\
\text { Brandegee }\end{array}$ & Zoë 5(10B): 202. 1905. & Actual & $\begin{array}{l}\text { Basónimo: Glycine } \\
\text { sagittata Humb. \& } \\
\text { Bonpl. ex Willd. }\end{array}$ \\
\hline 9 & Fabaceae & $\begin{array}{l}\text { Desmanthus } \\
\text { depressus } \\
\text { Humb. \& } \\
\text { Bonpl. ex Willd. }\end{array}$ & $\begin{array}{l}\text { Species Plantarum. } \\
\text { Editio quarta } 4(2): 1046 . \\
1806 .\end{array}$ & $\begin{array}{l}\text { Sinónimo de } \\
\text { Desmanthus } \\
\text { virgatus (L.) } \\
\text { Willd. }\end{array}$ & \\
\hline 10 & Fabaceae & $\begin{array}{l}\text { Mimosa debilis } \\
\text { Humb. \& } \\
\text { Bonpl. ex Willd. }\end{array}$ & $\begin{array}{l}\text { Species Plantarum. } \\
\text { Editio quarta } 4(2): 1029 . \\
1806 .\end{array}$ & Actual & \\
\hline 11 & Fabaceae & $\begin{array}{l}\text { Mimosa pellita } \\
\text { Humb. \& } \\
\text { Bonpl. ex Willd. }\end{array}$ & $\begin{array}{l}\text { Species Plantarum. } \\
\text { Editio quarta 4(2): } \\
\text { 1037-1038. 1806. }\end{array}$ & $\begin{array}{l}\text { Sinónimo de } \\
\text { Mimosa pigra L. } \\
\text { var. pigra }\end{array}$ & \\
\hline 12 & Fabaceae & $\begin{array}{l}\text { Mimosa } \\
\text { somnians } \\
\text { Humb. \& } \\
\text { Bonpl. ex Willd. }\end{array}$ & $\begin{array}{l}\text { Species Plantarum. } \\
\text { Editio quarta } 4(2): 1036 . \\
1806 .\end{array}$ & Actual & \\
\hline 13 & Gleicheniaceae & $\begin{array}{l}\text { Gleichenia } \\
\text { pubescens } \\
\text { (Humb. \& } \\
\text { Bonpl. ex } \\
\text { Willd.) Kunth }\end{array}$ & $\begin{array}{l}\text { Nova Genera et Species } \\
\text { Plantarum (quarto ed.) 1: } \\
\text { 29. } 1815 \text { [1816]. }\end{array}$ & $\begin{array}{l}\text { Sinónimo de } \\
\text { Sticherus bifidus } \\
\text { (Willd.) Ching }\end{array}$ & $\begin{array}{l}\text { Basónimo: } \\
\text { Mertensia } \\
\text { pubescens Humb. } \\
\text { \& Bonpl. ex Willd. }\end{array}$ \\
\hline
\end{tabular}




\begin{tabular}{|c|c|c|c|c|c|}
\hline $\mathbf{N}^{\circ}$ & Familia & Especie & Publicación & Status & Observación \\
\hline 14 & Moraceae & $\begin{array}{l}\text { Ficus prinoides } \\
\text { Humb. \& } \\
\text { Bonpl. ex Willd. }\end{array}$ & $\begin{array}{l}\text { Species Plantarum. } \\
\text { Editio quarta } 4(2): 1149 . \\
1806 .\end{array}$ & Actual & $\begin{array}{l}\text { Según la flora } \\
\text { del cono sur, } \\
\text { la variedad } \\
\text { subtriplinervia } \\
\text { es la que se } \\
\text { distribuye en } \\
\text { Paraguay y es } \\
\text { sinónimo de Ficus } \\
\text { pertusa L. f. }\end{array}$ \\
\hline 15 & Pteridaceae & $\begin{array}{l}\text { Adiantum } \\
\text { serrato- } \\
\text { dentatum } \\
\text { Humb. \& } \\
\text { Bonpl. ex Willd. }\end{array}$ & $\begin{array}{l}\text { Species Plantarum. } \\
\text { Editio quarta } 5: 445 . \\
1810 .\end{array}$ & Actual & \\
\hline 16 & Pteridaceae & $\begin{array}{l}\text { Adiantum } \\
\text { tetraphyllum } \\
\text { Humb. \& } \\
\text { Bonpl. ex Willd. }\end{array}$ & $\begin{array}{l}\text { Species Plantarum. } \\
\text { Editio quarta } 5(1): 441 . \\
1810 .\end{array}$ & Actual & \\
\hline 17 & Rubiaceae & $\begin{array}{l}\text { Machaonia } \\
\text { acuminata } \\
\text { Bonpl. }\end{array}$ & $\begin{array}{l}\text { Plantae Aequinoctiales } \\
\text { 1: } 101-103, \text { t. } 29.1808 \\
\text { [1806]. }\end{array}$ & Actual & \\
\hline 18 & Selaginellaceae & $\begin{array}{l}\text { Selaginella } \\
\text { marginata } \\
\text { (Humb. \& } \\
\text { Bonpl. ex } \\
\text { Willd.) Spring }\end{array}$ & Flora 21: 194. 1838. & Actual & $\begin{array}{l}\text { Basónimo: } \\
\text { Lycopodium } \\
\text { marginatum } \\
\text { Humb. \& Bonpl. ex } \\
\text { Willd. }\end{array}$ \\
\hline 19 & Solanaceae & $\begin{array}{l}\text { Datura } \\
\text { suaveolens } \\
\text { Humb. \& } \\
\text { Bonpl. ex Willd. }\end{array}$ & $\begin{array}{l}\text { Enumeratio Plantarum } \\
\text { Horti Botanici } \\
\text { Berolinensis, 227. } 1809 .\end{array}$ & $\begin{array}{l}\text { Sinónimo de } \\
\text { Brugmansia } \\
\text { suaveolens } \\
\text { (Humb. \& Bonpl. } \\
\text { ex Willd.) Sweet }\end{array}$ & \\
\hline
\end{tabular}

Tabla 2. Especies dedicadas a Aimé Bonpland presentes en Paraguay.

Table 2. Species dedicated to Aimé Bonpland present in Paraguay.

\begin{tabular}{|c|c|c|c|c|c|}
\hline $\mathbf{N}^{\circ}$ & Familia & Especie & Publicación & Status & Homenaje \\
\hline 1 & Araliaceae & $\begin{array}{l}\text { Hydrocotyle } \\
\text { bonplandii auct. non } \\
\text { A. Rich. }\end{array}$ & $\begin{array}{l}\text { Ann. Gen. Sci. } \\
\text { Phys. iv. } 192 \text {. t. } 54 \text {. } \\
\text { f. } 7.1820 .\end{array}$ & $\begin{array}{l}\text { Sinónimo de } \\
\text { Hydrocotyle filipes } \\
\text { Mathias }\end{array}$ & Epíteto \\
\hline 2 & Asparagaceae & $\begin{array}{l}\text { Herreria bonplandii } \\
\text { Lecomte }\end{array}$ & $\begin{array}{l}\text { Bull. Soc. Bot. } \\
\text { France 56: } 346 . \\
1909 .\end{array}$ & Actual & Epíteto \\
\hline 3 & Asparagaceae & $\begin{array}{l}\text { Herreria } \\
\text { montevidensis } \\
\text { Klotzsch ex Griseb. } \\
\text { var. bonplandii } \\
\text { (Lecomte) L.B. Sm. }\end{array}$ & $\begin{array}{l}\text { J. Wash. Acad. } \\
\text { Sci. 48: 282. } 1958 .\end{array}$ & $\begin{array}{l}\text { Sinónimo de } \\
\text { Herreria bonplandii } \\
\text { Lecomte }\end{array}$ & $\begin{array}{l}\text { Variedad, pero } \\
\text { originalmente } \\
\text { epíteto }\end{array}$ \\
\hline 4 & Bromeliaceae & $\begin{array}{l}\text { Billbergia } \\
\text { bonplandiana } \\
\text { Gaudich. ex Mez }\end{array}$ & $\begin{array}{l}\text { Flora Brasiliensis } \\
\text { 3(3): } 421 \text {, pl. } 76 . \\
\text { 1892. }\end{array}$ & $\begin{array}{l}\text { Sinónimo de } \\
\text { Billbergia nutans } \mathrm{H} \text {. } \\
\text { Wendl. ex Regel }\end{array}$ & Epíteto \\
\hline 5 & Cactaceae & $\begin{array}{l}\text { Harrisia bonplandii } \\
\text { (Pfeiff.) Britton \& } \\
\text { Rose }\end{array}$ & $\begin{array}{l}\text { Cactaceae (Britton } \\
\text { \& Rose) 2: 157, pl. } \\
\text { 24. 1920. }\end{array}$ & Actual & Epíteto \\
\hline 6 & Chloranthaceae & $\begin{array}{l}\text { Hedyosmum } \\
\text { bonplandianum } \\
\text { Kunth }\end{array}$ & $\begin{array}{l}\text { Nov. Gen. Sp. } \\
\text { (quarto ed.) } 7: 165- \\
\text { 166. 1825. }\end{array}$ & $\begin{array}{l}\text { Sinónimo de } \\
\text { Hedyosmum } \\
\text { brasiliense Miq. }\end{array}$ & Epíteto \\
\hline
\end{tabular}




\begin{tabular}{|c|c|c|c|c|c|}
\hline $\mathbf{N}^{\circ}$ & Familia & Especie & Publicación & Status & Homenaje \\
\hline 7 & Euphorbiaceae & $\begin{array}{l}\text { Croton } \\
\text { bonplandianus Baill. }\end{array}$ & $\begin{array}{l}\text { Adansonia 4: } 339 . \\
1864 .\end{array}$ & Actual & Epíteto \\
\hline 8 & Fabaceae & $\begin{array}{l}\text { Lupinus } \\
\text { bonplandianus C.P. } \\
\text { Sm. }\end{array}$ & $\begin{array}{l}\text { Spec. Lupinorum } \\
\text { 212. } 1940 .\end{array}$ & $\begin{array}{l}\text { Sinónimo de } \\
\text { Lupinus gibertianus } \\
\text { C.P. Sm. var. } \\
\text { gibertianus }\end{array}$ & Epíteto \\
\hline 9 & Lythraceae & $\begin{array}{l}\text { Cuphea bonplandii } \\
\text { Lourteig }\end{array}$ & $\begin{array}{l}\text { Lilloa 9: 346, fig. } 9 . \\
1943 .\end{array}$ & Actual & Epíteto \\
\hline 10 & Moraceae & $\begin{array}{l}\text { Sorocea bonplandii } \\
\text { (Baill.) W.C. Burger, } \\
\text { Lanj. \& Wess. Boer }\end{array}$ & $\begin{array}{l}\text { Acta Bot. Neerl. 11: } \\
\text { 465. } 1962 \text {. }\end{array}$ & Actual & $\begin{array}{l}\text { Epíteto, } \\
\text { originalmente } \\
\text { del género } \\
\text { Pseudosorocea }\end{array}$ \\
\hline 11 & Poaceae & $\begin{array}{l}\text { Digitaria bonplandii } \\
\text { Henrard }\end{array}$ & $\begin{array}{l}\text { Monogr. Digitaria } \\
80.1950 \text {. }\end{array}$ & $\begin{array}{l}\text { Sinónimo } \\
\text { de Digitaria } \\
\text { corynotricha (Hack.) } \\
\text { Henrard }\end{array}$ & Epíteto \\
\hline
\end{tabular}

\section{Discusión}

Es indiscutible que el gran legado dejado por Humboldt y Bonpland, fueron las colecciones de la biodiversidad presentes en los sitios en donde estuvieron. Las mismas representan las pruebas científicas de lo referido a ambos estudiosos, a la medida de cada uno, aunque en la obra "Vojage aux régions equinocciales du nouveau continent" apenas se tratan 148 especies de plantas (Prüfer Leske, 2019), de las más de 4.000 colectadas, aunque otras menciones hablan de que se entregaron al Museo de París 45 cajones que contenían más de 6.200 especies, representadas por unos 60.000 ejemplares (Bordas, 1990), con los duplicados incluidos. Este grandioso herbario fue confeccionado por Bonpland. La misma autora refiere que más de las $4 / 5$ partes fueron descritas por Bonpland. Tampoco puede discutirse el mérito intelectual, reflejado en los escritos conjuntos de cada uno de ellos, pues gracias a éstos se ha accedido y se accede al conocimiento.

Al botánico A. Bonpland, ¿cómo se lo valora en su real medida? Es muy difícil poder hacerlo si los críticos a su quehacer como botánico y zoólogo de campo no han vivido lo que el naturalista experimentó. El trabajo de campo es muy duro y sin tiempo, trabajar a la intemperie, bajo el sol, con humedad o viento, expone al botánico a un cansancio muy particular. Muchas veces el acceso a las plantas no es muy fácil y ocasiona el extravío del botánico, entre otras vicisitudes y las plantas no siempre son fáciles de obtener. Las colecciones de campo, luego de un largo día de colecta requieren un trabajo posterior: acomodarlas de manera que queden buenas para ser estudiadas, secarlas y tratarlas obligatoriamente in situ, separarlas para su distribución, ficharlas una a una y ese proceso no espera, se lo debe hacer a medida que las mismas van siendo colectadas y procesadas, entre otras actividades obligatorias. De manera que la tarea no se circunscribe solo a unas pocas horas de trabajo diario sino a todo un día con parte y a veces gran parte de la noche. Un trabajo abrumador para más de las 4.000 especies que han sido colectadas y cuya responsabilidad prácticamente recayó sobre Bonpland.

No obstante, en los primeros tiempos realizó un trabajo de taxónomo describiendo especies para la ciencia, trabajo inconcluso y que lo completó posteriormente Carl Sigismund Kunth, botánico alemán quien publicó las nuevas especies descriptas de las colectadas por Humboldt y Bonpland en su Nova Genera et Species Plantarum en 7 tomos escritos por él sobre las colecciones mencionadas.

Obviamente, los logros del joven Kunth, quien se dedicó de lleno a los estudios de las colecciones herborizadas, fueron abrumadores, tarea que le llevó muchos años. Como lo menciona Prüfer Leske (2019), las nuevas plantas descriptas enriquecieron en gran medida a la botánica, lo que mejoró sustancialmente el conocimiento de la flora tropical americana, destacando a su vez las cualidades de Kunth 
para el trabajo en gabinete, cualidad de la que, según la misma autora, Bonpland carecía.

Lo cierto es que el trabajo de Kunth tal vez no hubiera tenido el mismo impacto, si las colecciones que sirvieron de base para tantas descripciones de especies nuevas para la ciencia no hubieran venido del nuevo mundo. Creemos que es así como se deben balancear los resultados y no separar las actividades del equipo. No se va a juzgar en este trabajo la denominación única de Kunth como único autor de los nombres de las especies descriptas porque es absolutamente correcto, solo se pretende señalar cuán injusta es la ciencia cuando desaparecen los nombres de quienes en primera instancia muy probablemente ya sabían que dichas especies correspondían a nuevos descubrimientos para la ciencia, pero no pudieron culminar el proceso.

La denominación original a las descripciones realizadas por Kunth fue H. B. K., aludiendo a Humboldt, Bonpland y Kunth, lo cual demuestra la admiración y porque no, el agradecimiento de este joven botánico hacia los dos maestros. Es posible que una mejor denominación autoral hubiera sido $\mathrm{K}$. B. \& H., aludiendo a Kunth como primer autor, Bonpland como el segundo por haber sido el botánico y Humboldt el tercero y que probablemente fue el que observó y describió cada uno de los hábitats de las mismas, como base para su destacado trabajo de fitogeografía americana. Como muy bien lo menciona Prüfer Leske (2019), lo importante no es discriminar entre los autores que porcentaje de responsabilidad le correspondió a cada uno, sino recordar que, gracias a los tres, salieron las grandes obras reconocidas hoy día.

Ya en América y valorando el mérito de taxónomo, se puede destacar que en su cuaderno botánico "Journal de Botanique" (Bonpland, 1924), se encuentra que Bonpland demostró especial interés en la taxonomía del género Ilex y describió I. theazans, cuyo nombre no fue aplicado a la misma entidad, razón por la que no fue reconocido. Estos escritos fueron muy estudiados por Giberti (1988, 1990, 2011). El mismo autor menciona que en sus cuadernos de botánica se encontraron otras descripciones del género Ilex tales como I. brevifolia, I. crepitans, I. gigantea, I. humboldtiana, las que permanecieron inéditas hasta ser publicadas a partir de la década de 1860 e inclusive hasta después de la muerte del botánico.

Entre sus apuntes no solo había descripciones muy bien detalladas de las especies que le interesaban, sino también dibujos, los que ayudaron a una mejor descripción de Victoria cruziana, "yacaré yrupé" o "maíz del agua", encontrada en los alrededores de Corrientes (Argentina) y descripta primero con el nombre genérico de Nymphaea, lo que fue corregido gracias a las excelentes descripciones de Bonpland.

El nivel de detalle de los cuadernos de estos naturalistas debía ser muy minucioso, ya que de eso dependía, mucho o completamente la determinación posterior, por la falta de material bibliográfico. No obstante de que lo tenían consigo, en algunos casos eran víctimas de robo, como lo mencionó un amigo y colega de Bonpland, el suizo Johann Rengger, quien también estuvo retenido en Paraguay hasta el año 1825. El mismo no podía realizar determinaciones de sus colecciones debido a que los escritos histórico-naturales que había traído de Europa le habían sido sustraídos en Buenos Aires, por esto se dedicó más bien en describir de manera precisa a las variedades, dejando su determinación para el futuro (Rengger, 2010).

A propósito de sus trabajos taxonómicos, los que se inician por cierto con las colecciones botánicas, Cerruti $(2003,2019)$ menciona que en América, Bonpland llevaba una colección de herbario de más de 20.000 plantas, semillas, entre otras, antes de su confinamiento en Misiones, Paraguay. Este número es muy elevado y no se especifica en cuánto tiempo lo colectó, si realizó expediciones acompañado por otras personas que le hubieran ayudado en la tarea de colecta, como tampoco se esclarece muy bien si esa cantidad de números colectados fueron de plantas originales o si en dicha colección estaban considerados ya los duplicados, teniendo en cuenta que él enviaba sus plantas al "Musée d'Histoire Naturelle", París. Otros datos proporcionados por el mismo autor quien menciona que en los escritos posteriores de Bonpland se registran 2.884 números de plantas colectadas $\mathrm{y}$ en sus cuatro cuadernos de colecciones en 
Sudamérica, se menciona que entre 1817 y 1849 existen especímenes enumerados desde el 1 al 2.449, lo cual es mucho más realista que los números mencionados por Cerruti (2019).

Durante su confinamiento en Paraguay, que duró más de nueve años, algunos mencionan que realizó colecciones en el país (Lourteig, 1977; Schinini et Arbelo de Mazzaro, 2008), agregan que lo hizo incansablemente, pero sin dar mayores detalles. De acuerdo a Mereles (2008), él era un botánico de corazón y no hubiera dejado pasar tantas oportunidades de realizarlas, por lo cual se puede inferir que cuando salió de su confinamiento, llevó colecciones de herbario del país e igualmente cuando retornó a Paraguay luego de la muerte del dictador don Rodríguez de Francia en el año 1857, las volvió a realizar (Contreras Roqué et Boccia Romañach, 2006).

Como prueba de su interés como taxónomo, se menciona una traducción de Lourteig (1977), que en el año 1831 y ya saliendo de su confinamiento involuntario, colectó una planta, mencionando lo siguiente: "durante 6 años consecutivos no dejé de hacer investigaciones sobre esta curiosa planta y no es desde hace poco que he podido conocer su localidad, estudiarla y describirla de manera exacta..." (sic). Se trató de Lophophytum sp., Balanophoraceae, una especie parásita de las raíces de algunos árboles tales como Enterolobium, Inga, Piptadenia y Pithecelobium (Hansen, 1987), especie muy bien distribuida en los bosques de la región Oriental del país, que fue descripta en 1832 .

Los escritos demuestran otra tendencia o gusto de A. Bonpland por las plantas y ésta trata del uso que se daba a las mismas, lo que hoy llamamos etnobotánica, botánica económica, u otra denominación similar, es decir la aplicación directa de las especies por el hombre. Según Arbelo de Mazzaro (2013), en Quito se encuentra un libro donado por el mismo Bonpland, que contiene 135 dibujos de plantas, algunas medicinales y otras útiles, y les designa a todas, un nombre en latín y otro en lengua guaraní.

No fue solo en Sudamérica sino ya en París, cultivó especies útiles y ornamentales en los jardines de la Malmaison (Lourteig, 1977; Contreras Roqué et Boccia Romañach, 2006;
Arbelo de Mazzaro, 2019). En efecto, en dicho jardín parisino, se dedicó a las tareas de horticultura y floricultura, en donde incluyó a varias especies exóticas de interés no solo decorativo sino alimenticio, entre otros usos, lo cual indica una inclinación e interés muy fuerte hacia el uso de las plantas. Rossi (2019) menciona que esta actividad fue realizada innovando con el uso de invernaderos y viveros hasta 1814, e inauguró en dicho jardín una nueva estética revolucionaria neoclásica, que posteriormente se expande por toda Europa. También se debe mencionar su permanente interés por la "yerba mate", que denota en sus estudios taxonómicos que tienen directa relación más con el uso de la especie que con un interés taxonómico propiamente dicho.

En su lugar de confinamiento en Santa María de Fe, Paraguay, aprendió probablemente de las mujeres rurales e indígenas de la zona en donde vivió, las cualidades de varias plantas utilizadas como medicinal, las que cultivó y posteriormente le sirvieron para el tratamiento de sus pacientes pues Bonpland prestaba servicio como médico. El caso de la yerba mate, Ilex paraguariensis A. St.-Hil., motivo de su apresamiento, enseñó como una novedad el cultivo de la misma a la población rural, emulando un tanto el ejemplo que dejaron los Jesuitas con los indígenas en las Reducciones Jesuíticas, transformando esta actividad en Argentina como un modelo de explotación (Espinosa, 1997).

Un dato no menos importante es el que menciona Cerruti (2019), acerca del deseo de Bonpland quien se sentía motivado de trabajar para el interés general y ambicionó hacer un establecimiento de agricultura para multiplicar las plantas indígenas y exóticas útiles para repartirlas por toda la provincia.

$\mathrm{Su}$ inclinación por el uso de las plantas fue tal que también se interesó en la Victoria cruziana Orb., el "yrupé”, "yacaré yrupé” o el "maíz del agua" como se la conoce, por considerar que las semillas podrían tener un uso alimenticio (aparte de ser una planta acuática muy ornamental).

Igualmente, cultivaba especies silvestres que poseen rizomas, probablemente con interés alimenticio, pero también con fines medicinales, como el caso de algunas especies 
de Jatropha (Euphorbiaceae), que crecían en los alrededores (Mereles, 2008).

Es muy posible que Bonpland, confinado en Santa María de Fe no tuviera otra opción que dedicarse de lleno a las actividades de campo, como tampoco negar el hecho de ir descubriendo las cualidades de las especies locales para diversos usos. Tarea apasionante, en un medio en donde ya la población desde los tiempos de los guaraníes, mantenían la costumbre de alimentarse y curar sus enfermedades con los recursos existentes en el medio natural.

Por todos los logros como botánico y naturalista, obtuvo reconocimiento de diversas maneras por parte de la comunidad botánica, dedicándoles especies. Otros han recopilado las dedicatorias (Schinini et Arbelo de Mazzaro, 2008) en donde incorporaron especies de varias partes, al igual que este trabajo, que concentró a especies que llegan a Paraguay.

\section{Conclusiones}

Se ha hablado y escrito bastante sobre Aimé Bonpland desde prácticamente el siglo XIX e inicios del siglo XX hasta la actualidad (Delessert, 1854; Hamy, 1906; Bonpland, 1924; Castellanos, 1963; Lourteig, 1977; Boccia Romañach, 1999; Schneppen, 2000; Hossard, 2001; Contreras Roqué et Boccia Romañach, 2006; Krapovickas, 2008; Bell, 2010; Giberti, 2011; Cerruti, 2019; entre otros), debido a que este hombre ha tenido múltiples facetas en su vida, como lo afirmaran varios de los autores mencionados.

No se conocen a cabalidad las publicaciones científicas realizadas durante su estadía en el nuevo mundo, según lo manifiestan algunos autores (Castellanos, 1963; Lourteig, 1977), excepto algunos manuscritos realizados sobre la "yerba mate", Ilex paraguariensis (Giberti, 2011). Sin dudas, su trabajo merece ser estudiado, para conocer realmente su legado. A pesar de que Bonpland no ocupaba su tiempo en publicar sus estudios, fue muy minucioso con los registros de sus observaciones en sus diarios de viajes, notas, listado de especies, incluso observaciones meteorológicas (Giberti, 2008).
Lamentamos que las colectas realizadas en Paraguay durante su confinamiento de más de nueve años, así como las realizadas a su regreso a Asunción en 1857, no estén listadas porque para el país constituirían las primeras colecciones científicas de la flora paraguaya con respaldo de material de herbario, inclusive primero que aquellas realizadas por el Dr. J. Rengger en la década de 1820 al 30.

Finalmente, a decir de Sanjurjo (2019), el Paraguay está en deuda con Bonpland y que las rememoraciones sucesivas que se dieron, aunque escasas, ayuden a ponerlo en el pedestal que corresponde.

\section{Agradecimientos}

Los autores agradecen a los Herbarios FCQ, PY y SCPY por permitirnos la revisión de los especímenes de la flora paraguaya mencionada en las determinaciones taxonómicas realizadas por A. Bonpland y el listado de las especies que les fueron dedicadas.

\section{Bibliografía}

ARBELO DE MAZZARO, A. (2013). El legado de Amado Bonpland en Corrientes. Revista Fuentes 7: 29-42.

ARBELO DE MAZZARO, A. (2019). Abordaje de la construcción cultural-natural de los poblados visitados por Bonpland durante sus recolecciones científicas en Corrientes y Paraguay. En ARBELO DE MAZZARO, A., G. D. MANGO DE RUBIO \& J. A. PERSI (eds.), II Congreso Internacional Interdisciplinario Aimé Bonpland-Alexander von Humboldt Año 2017, pp. 201-224. Instituto de Cultura, Moglia Ediciones, Corrientes.

BECK, H. \& SCHOENWALT, P. (1999). El último de los grandes: Alexander von Humboldt. Contornos de un genio. Inter Nationes, Bonn.

BELL, S. (2010). A life in shadow. Aimé Bonpland in Southern South America 1817-858. Stanford University Press, California.

h t t p s : // d o i.org/1 0 . $111226 /$ stanford/9780804752602.001.0001

BOCCIA ROMAÑACH, A. (1999). Amado Bonpland, Caraí Arandú. Ed. El Lector, Asunción-Paraguay. 
BOCCIA ROMAÑACH, A. (2001). El Polifacético Aimé Bonplad. Fundación Félix de Azara, Ser. Ciencia y Técnica 1: 1-14.

BONPLAND, A. (1924). Bonpland, A. (1924). Archives Inédites. Journal de Botanique, Tomo 2. Trabajos del Instituto de Botánica y Farmacología, Facultad de Ciencias Médicas de Buenos Aires, 42. Talleres S. A. Casa Jacobo Pauser, Buenos Aires.

BORDAS, E. (1990). El Naturalista Bonpland. La Revista Crítica 1: 40-43.

CASTELlANOS, A. (1963). Bonpland en los países del Plata. Revista de la Academia de Ciencias Exactas de Colombia 12: 57-86.

CERRUTI, C. (2003). Aimé Bonpland, botanist et avénturieur. La Revue 39/40: 38-47.

CERRUTI, C. (2019). Aportes Científicos de Aimé Bonpland a las ciencias en América como adhesión a los 200 años de su regreso a estas tierras. En ARBELO DE MAZZARO, A., G. D. MANGO DE RUBIO \& J. A. PERSI (eds.), II Congreso Internacional Interdisciplinario Aimé Bonpland-Alexander von Humboldt Año 2017, pp. 19-40. Instituto de Cultura, Moglia Ediciones, Corrientes.

CONTRERAS ROQUÉ, R. \& BOCCIA ROMAÑACH, A. (2006). El Paraguay en 1857. Un Viaje Inédito de Aimé Bonpland. Universidad Nacional de Pilar y Editorial ServiLibro, Asunción, Paraguay.

DELESSERT, F. (1854). Communication faite par M. Francois Delessert d'une lettre de M. de Bonpland. Comptes Rendus Hebdomadaires des Séances de l'Académie des Sciences 38: 434-438.

ESPINOSA, N. C. (1997). Amado Bonpland. Una historia olvidada. Ediciones Colmegna, Santa Fe.

GIBERTI, G. C. (1988). Aquifoliaceae paraguayas. Notas críticas en el género Ilex. En SPICHIGER, R. (ed.), Notulae ad Floram paraquaiensem pp. 14-17. Candollea 43: 417- 420.

GIBERTI, G. C. (1990). Bonpland's manuscript name for the Yerba Mate and Ilex theezans C. Martius ex Reisseck (Aquifoliaceae). Taxon 39: 663-665. https://doi.org/10.2307/1223390

GIBERTI, G. C. (2008). El Archivo Bonpland en el Museo de Farmacobotánica "Juan Aníbal Domínguez". Dominguezia 24: 5-9.

GIBERTI, G. C. (2011). La "Yerba Mate" (Ilex paraguariensis, Aquifoliaceae) en tempranos escritos rioplatenses de Bonpland y su real distribución geográfica en Sudamérica Austral. Bonplandia 20: 203-212. https://doi.org/10.30972/ bon. 2021324
HAMY, T. J. E. (1906). Aimé Bonpland, médicin naturaliste explorateur de l'Amerique du Sud, sa vie, sa correspondece. Librarie Orientale \& Américaine. E. Guilmoto, París.

HANSEN, B. (1987). Balanophoraceae. En SPICHIGER, R. (ed.), Flora del Paraguay. Conservatoire et Jardin Botaniques de la Ville de Genéve \& Missouri Botanical Garden.

HOSSARD, N. (2001). Aimé Bonpland (1773-1858), médicin, naturaliste explorateur en Amérique du Sud. Á l'ombre des arbres. L'Harmattan, París.

KRAPOVICKAS, A. (2008). Bonpland, sesquicentenario de su muerte. Bonplandia 17: 5-11.

https://doi.org/10.30972/bon.1711365

LOURTEIG, A. (1977). Aime Bonpland. Bonplandia 3: 269-317. https://doi.org/10.30972/bon.3162590

MERELES, F. (2008). El legado científico de Aimé Bonpland. La Revista Crítica 18: 45-50.

PRÜFER LESKE, I. (2019). La recepción actual de Alexander von Humboldt y Aimé Bonpland, análisis y comparativo. En ARBELO DE MAZZARO, A., G. D. MANGO DE RUBIO \& J. A. PERSI (eds.), II Congreso Internacional Interdisciplinario Aimé Bonpland-Alexander von Humboldt Año 2017, pp. 67-98. Instituto de la Cultura, Moglia Ediciones, Corrientes.

RENGGER, J. R. (2010). Viaje al Paraguay en los años 1818 a 1826. En TOMASINI, A. \& J. BRAUNSTEIN (traductores). Tiempo de Historia, Asunción [Traducción al español de J. R. RENGGER (1835)].

ROSSI, L. (2019). La geopolítica discursiva de Bonpland: itinerarios y consecuencias de sus "herborificaciones". En ARBELO DE MAZZARO, A., G. D. MANGO DE RUBIO \& J. A. PERSI (eds.), II Congreso Internacional Interdisciplinario Aimé Bonpland-Alexander von Humboldt Año 2017, pp. 41-52. Instituto de la Cultura, Moglia Ediciones, Corrientes.

SANJURJO, M. (2008). Recordando a A. Bonpland en Paraguay. La Revista Crítica 18: 31-44.

SANJURJO, M. (2019). Recordando a Bonpland. En ARBElO DE MAZZARO, A., G. D. MANGO DE RUBIO \& J. A. PERSI (eds.), II Congreso Internacional Interdisciplinario Aimé BonplandAlexander von Humboldt Año 2017, pp. 277305. Instituto de la Cultura, Moglia Ediciones, Corrientes.

SCHININI, A. \& ARBELO de MAZZARO, A. (2008). Especies dedicadas a Amado Bonpland. 2008, Año Bonplandiano. 1858-2008 sesquicentenario 
del fallecimiento del Dr. Amado Bonpland. SubSecretaría de Cultura, Corrientes.

SCHNEPPEN, H. (1999). Aimé Bonpland, el olvidado o compañero de Humboldt? Anuario de la Academia Paraguaya de la Historia 39: 48-56.

SCHNEPPEN, H. (2000). Aimé Bonpland - Alexander von Humboldt vergessener Gefahrte. Alexander von Humboldt - Forschungsstelle, Berlín.

THIERS, B. (2020). Index Herbariorum: a global directory of public herbaria and associated staff. New York Botanical Garden's Virtual Herbarium, http://sweetgum.nybg.org/ih 
